\title{
Reviewing the potential use of scaffold-mediated localized chemotherapy in oncology
}

Research Article

Archana A Gupta ${ }^{1}$, Supriya Kheur ${ }^{1 *}$, A. Thirumal Raj², Ravindra V. Badhe ${ }^{3}$, Ramesh. R. Bhonde 4

${ }^{1}$ Assistant Professor, Department of Oral Pathology and Microbiology, Dr. D.Y Patil Dental College and Hospital, Dr. D.Y Patil Vidyapeeth, Pune, Maharashtra-411041, India

${ }^{2}$ Department of Oral Pathology and Microbiology, Sri Venkateswara Dental College and Hospital, Thalambur, Chennai-600130, India ${ }^{3}$ Department of Pharmaceutical Sciences and Research, Dr. D.Y Patil College of Pharmacy, Dr. D.Y Patil Vidyapeeth, Pune, Maharashtra-411041, India ${ }^{4}$ Research Director, Dr. D.Y Patil Vidyapeeth, Pune, Maharashtra-411041, India

Received 25 April 2019; Accepted 11 December 2019

\begin{abstract}
Post-surgical recurrence and metastasis remain to be the major concern in oncology. The absence of any therapeutic modality during the interim period between the surgical intervention and initiation of conventional radiotherapy and chemotherapy allows the residual cancer cells to proliferate, culminating in recurrence and/or metastasis. Introducing a therapeutic modality during this interim period could suppress the proliferation of the residual tumor cells. Further, as the detrimental effects of conventional chemotherapy and radiotherapy drastically reduce the patient's quality of life, use of therapeutic modality with localized effect can reduce the risk of systemic toxicity. Thus, the present manuscript reviews the potential use of scaffold-mediated local chemotherapy in oncology. Its localized effect would prevent systemic toxicity, while the scaffold serves as an ideal vehicle for the sustained targeted delivery of therapeutic agents.
\end{abstract}

Keywords: Chemotherapy • Localized • Oncology • Scaffold • Tissue Engineering

\section{Introduction}

Locoregional recurrences (LRR), posing an interdisciplinary therapeutic challenge, are considered as a major cause for the poor survival rate in cancer $[1,2]$. Risk factors involved in LRR mostly include lymphovascular invasion, extracapsular spread, and distant metastasis [3] which often occurs within the first 24 months post-surgical intervention [4]. Adjuvant chemotherapy is generally started within 1 month (taken as an average time period of all the commonly occurring cancers including oral squamous cell carcinoma, breast cancer, prostate cancer, lung cancer, etc.) and radiotherapy is delayed until initial chemotherapy is completed [5-7]. This interim period of 4-5 months post-surgical excision allows proliferation of the residual tumor cells. The transient immune suppression following the surgical intervention provides an ideal environment for recurrence [8].

Although several studies have explored the potential use of scaffold-mediated drug delivery, there has been a lack of accuracy as to the dosage and duration of drug delivery. The timing of the drug delivery is very critical as in the case of the interim period wherein the residual tumor cells are allowed to grow due to the lack of any active therapeutic interventions. Administering the therapeutic agent at this time frame (interim period) would reduce the risk of LRR. In addition to the time of delivery, importance must be given to the delivery target. Nonspecific therapeutic modalities can lead to systemic toxicity. Thus, scaffold-mediated local chemotherapy (SMLC) could serve as an ideal therapeutic modality in the interim period due to its sustained targeted effect [9]. The present review focuses on the potential use of 
SMLC as a post-surgical treatment modality during the interim period in cancer.

\section{Tissue-engineered scaffolds for cell/drug delivery}

The past two decades have seen extensive research and development in the field of tissue engineering [10]. Tissue engineering involves the replacement and regeneration of diseased and damaged tissues. Commonly used substitutes for repairing damaged and diseased parts are autografts, allografts, and xenografts. Problems encountered with these substitutes, including donor shortage and graft rejection, have prompted researchers to investigate alternative sources [11]. Naturally derived and synthetically manufactured scaffolds are one such alternative which is studied extensively as both cell and drug delivery systems. Different cell/drug delivery scaffolds available are broadly classified as (1) implantable including 3D porous structures consisting of interconnected porosities allowing for good cell seeding density, and hence, tissue ingrowth in the form of matrix [12] and nanofibrous matrix fabricated through electrospinning simulating physiological environment [13] and (2) injectable comprising thermosensitive solgel transition hydrogel [14] and porous microspheres [15]. Tissue-engineered scaffolds should allow the basic interaction of components, including holding the cells and growth factors together and supporting the tissue matrix laid down by the cells. The ideal properties which these scaffold materials should possess include (1) biocompatibility, (2) optimal biodegradability, and (3) ideal mechanical properties [16-19]. Good surface to volume ratio and proper interface adherence would aid in the optimal drug/cell adherence and cell proliferation, facilitating cell migration $[17,18]$. Adequate porosities and their interconnectivity will allow the interaction of the drug and the target cell and aid in maintaining healthy vasculature around the scaffold materials [16,17]. It should be easily processed, should mimic the native extracellular matrix, and should possess optimum loadbearing and release kinetics for the cells and drugs incorporated $[18,20]$.

\section{Design strategies of SMLC}

Two major patterns of scaffold placement include injection-based and implant-based scaffold. Due to the surgical intervention of the latter, the injection-based implants are preferred. In addition to this, the pore size, interconnectivity, and high surface to volume ratio can be controlled optimally in injectable scaffolds. Different design strategies for scaffold fabrication have been discussed below:

1. Hydrogel-based systems: They include watersoluble polymers which can include both natural (collagen, chitosan, gelatine) and synthetic (polyethylene glycolide and polyvinyl alcohol) polymers. They can be cross-linked physically or chemically. The cross-linked polymers convert to gel form on exposure to water [21,22]. The polymers are biodegradable and their release kinetics can be controlled by a particular type and amount of crosslinking [23]. They exhibit a sol state at the room temperature, which changes into gel form at body temperature [18]. Their ease of administration and controlled release kinetics make them a potential cell/drug delivery carrier [24].

2. Microparticle-based system (microspheres): Microparticles are used as injectable scaffolds and possess sufficient porosities for effective cell/drug seeding, growth factors incorporation, and tissue ingrowth [18,24]. Nanofabricated microparticles show added advantages of regulation of properties like angiogenesis and cell migration [24]. Chitosan scaffolds in gelatine microspheres incorporated with fibroblast growth factors have been studied in wound closure [25].

3. Membrane-based system: Fabrication of bilayered structure membrane along with growth factors has been developed as an effective alternative for skin grafts, overcoming the complications of a shortage of donor sites, donor site morbidity, and graft reactions $[26,27]$. Incorporation of antibiotics along with growth factors in membrane-based systems helps in maintaining a sustained drug concentration at the wound site, preventing any risk of bacterial infection [28].

Tissue-engineered SMLC involves several methods of fabrication. Simplest one is the immersion of scaffold materials in drug solution, and the second one is by dissolving both the polymer and drug in the solvent. The release of drug from scaffolds in both the above techniques depends upon the rate of degradation of the scaffold and the amount and size of porosities present in the scaffold material. Some recent fabrication techniques involve the layer by layer technique, wherein the drug is incorporated between the layers and with $3 D$ printing technology, the shape, size, and architecture of the scaffold can be customized according to the specific need, along with the optimization of the dosage and location of the drug [29] (refer Figure 1). 


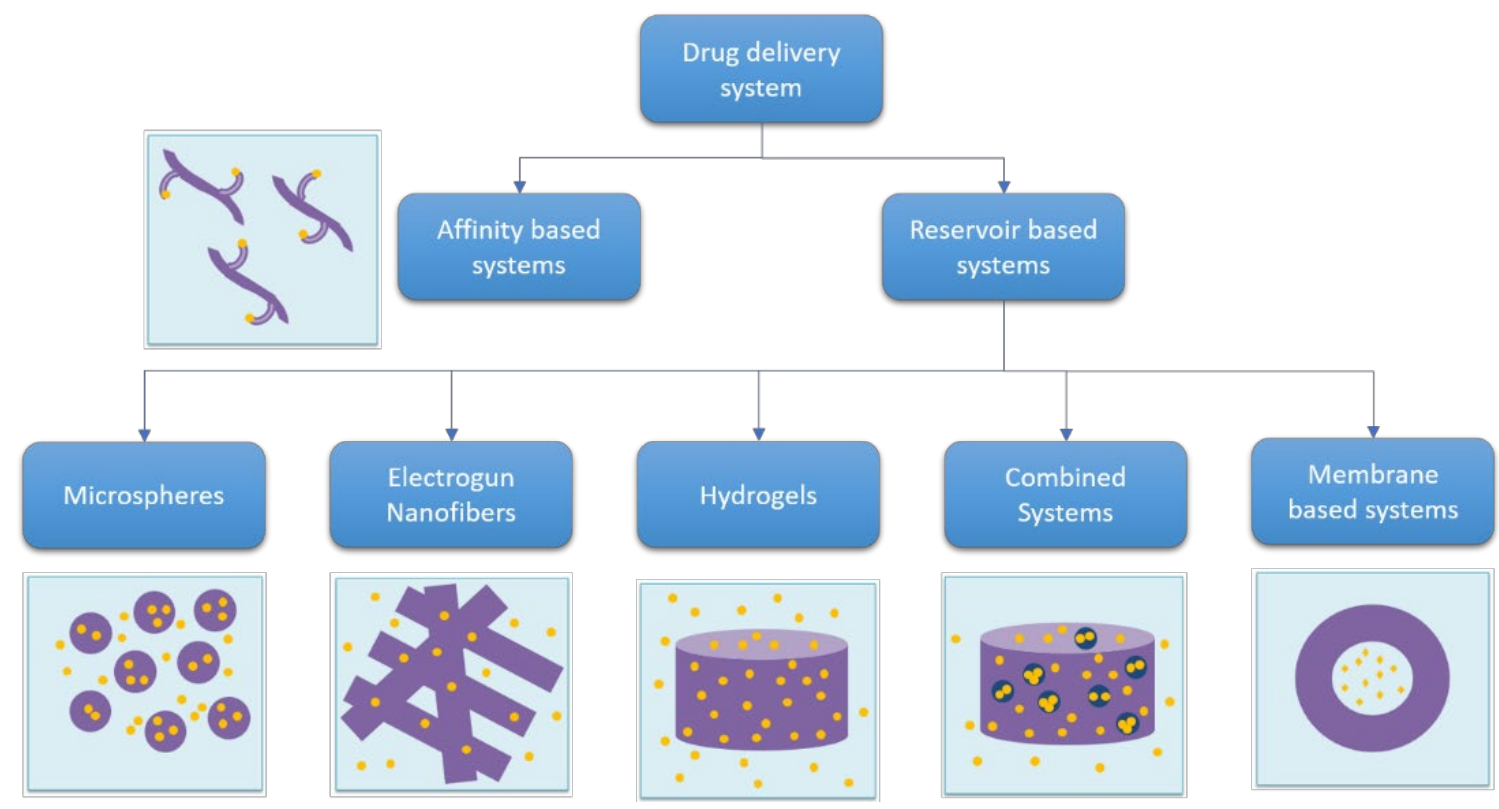

Figure 1: Different design strategies used for scaffold fabrication for scaffold-mediated localized chemotherapy.

\section{Recent advancements in SMLC}

With the above-discussed scaffold materials and techniques, extensive research has been carried out in the field of SMLC. However, several new and advanced customized techniques have also been discovered in recent years to increase the effectiveness of SMLC. Mesoporous silicate nanoparticles (MSNs) (mesoporous material refers to a material containing pores of diameter 2-50 nm) incorporated with 3D nanofibrous gelatine scaffolds have been prepared for dual delivery of growth factors including bone morphogenic protein 2 (BMP2) and deferoxamine (DFO). DFO activates hypoxiainducible factor 1a (HIF-1a), which further leads to angiogenesis which is essential for osteogenesis. The sustained release of BMP2 and HIF-1a is maintained by their covalent linking to the polymeric scaffold material, chitosan [30].

A nanostructured graphene polymer composite acting as the reinforcing agent to increase the strength of biodegradable scaffolds has been used for targeted drug delivery to cancer cells. Graphene also promotes adhesion and proliferation of cells on the scaffold surface, along with increasing its biological properties. Such scaffolds incorporated with sufficient amount of porosities are prepared with magnetically controlled 3D printing technology [31].

Polylactic acid (PLA) nanofibers are incorporated with natural pearl powder and doxorubicin hydrochloride
(DOX). This drug-loaded nanofibrous composite scaffold (DOX-PLA/Pearl) combines the osteoconductivity of pearl powder with the release of doxorubicin. The drug delivery rate increases as the amount of pearl powder increases, further increasing the antitumor efficacy of the scaffold material [32].

Biocompatible electrospun (fabrication method which draws charged threads of polymers using electric force to fabricate fibers of $100 \mathrm{~nm}$ diameter) nanofibrous scaffolds (bENS) are used to suppress recurrence of post-surgical glioblastomas by delivering cytotoxic stem cells. They help in the retention and persistent release of mesenchymal stem cells, compared to standard direct injection [33].

Hydroxycamptothecin (HCPT)-gold nanoparticle (AuNP) conjugates were tested in different sizes for their antitumor cytotoxic effect. Researchers have reported a notable cytotoxic effect of the conjugate on the MDAMB-231 cell line (breast cancer cell line) compared with an equal dose of free HCPT [34].

\section{Future prospects}

The future of scaffold-mediated localized delivery of cells, drugs, growth factors, and genes in tissue engineering lies in the fact that the release of materials incorporated in the scaffolds can be controlled, optimized, and customized for specific treatment. Authors have hypothesized the use of scaffolds for 
localized delivery of bone modulators for faster bone remodeling. Researchers are also trying to incorporate natural products in the scaffold materials, including plants of medicinal value. However, their controlled release will again remain a challenge for the researchers. Microfluidics technology and injectable gel-sol scaffolds find immense scope in the field of tissue engineering owing to their ease of use. Composite polymers made by combining the biodegradable natural polymers and high-strength synthetic polymer could be the future of scaffold materials. Incorporation of stem cells or osteoblastic cell lines along with drugs to help in both regeneration of post-surgical bone defects and localized delivery of chemotherapeutic holds immense potential in the field of oncology [35-38].

Use of scaffolds and hydrogel-based systems to boost the immune response of individuals for obtaining therapeutic benefit in cancer is also gaining importance these days [39].

Novel therapies against tumor-specific proteins such as Endothelial Growth Factor Receptor (EGFR) or human epidermal growth factor receptor 2 (Her2) with highly selective anticancer agents are the need of the hour. Mini-proteins and multicyclic peptides as scaffolds find their applications in precise diagnosis and targeted cancer treatment [40].

\section{References}

[1] Wadasadawala T, Vadgaonkar R, Bajpai J. Management of Isolated Locoregional Recurrences in Breast Cancer: A Review of Local and Systemic Modalities. Clin Breast Cancer. 2017 Nov; 17 (7): 493-502.

[2] Harms W, Geretschläger A, Cescato C, Buess M, Köberle D, Asadpour B. Current Treatment of Isolated Locoregional Breast Cancer Recurrences. Breast Care 2015; 10: 265-271.

[3] Elsayed M, Alhussini M, Basha A and Awad A. T. Analysis of loco-regional and distant recurrences in breast cancer after conservative surgery. World Journal of Surgical Oncology (2016) 14:144

[4] Van Tienhoven G, Voogd AC, Peterse JL, et al.: Prognosis after treatment for locoregional recurrence after mastectomy or breast conserving therapy in two randomized trials (EORTC 10801 and DBCG-82TM). EORTC Breast Cancer Cooperative Group and the Danish Breast Cancer Cooperative Group. Eur J Cancer 1999,35: 32-38.

[5] Karlsson P, Cole BF, Price KN, Gelber RD, Coates AS, Goldhirsch A. Timing of radiotherapy and chemotherapy after breast-conserving surgery for node-positive breast cancer: long-term results

\section{Conclusion}

The factors to be considered in formulating a scaffoldmediated drug delivery include host-scaffold interaction, cell/drug adherence, angiogenesis, and biodegradability of the scaffold material. The scaffold-host tissue interaction depends on the biocompatibility of the scaffold material and the design of the scaffold. The design must permit effective drug delivery without compromising the physical properties of the scaffold. Adherence of cells and drugs on the scaffold surface is vital, as lack of optimal adherence could result in premature cell/ drug release. Angiogenesis in and around the inserted scaffolds is vital to ensure the successful retention and functioning of the scaffold. The degradation rates of scaffolds should be customized such that they correspond to the intended dosage and duration of drug delivery. To conclude, SMLC holds a promising future in oncology if combined with the appropriate dosage and delivery time. Introducing SMLC in the interim period immediately following post-surgical intervention could reduce the risk of LRR, while its localized targeted effect prevents the risk of systemic toxicity.

from IBCSG Trials VI and VIIInt J Radiat Oncol Biol Phys. 2016 October 1; 96(2): 273-279

[6] Liu Y, Zhai X, Li J, Li Z, Ma D and Wang Z. Is there an optimal time to initiate adjuvant chemotherapy to predict benefit of survival in non-small cell lung cancer? Chin J Cancer Res. 2017; 29(3): 263-271

[7] Chay C, Smith DC. Adjuvant and neoadjuvant therapy in prostate cancer. Semin Oncol. 2001; 28(1): 3-12.

[8] Dąbrowska A M, Słotwiński R. The immune response to surgery and infection. Centr Eur J Immunol 2014; 39 (4): 532-537

[9] Gupta AA, Pande N, Kheur S, Raj AT. Assessing the potential role of scaffold-mediated local chemotherapy in oral cancer. Oral Oncol. 2018. https://doi.org/10.1016/j.oraloncology.2018.03.009

[10] Kretlow JD, Mikos AG. From material to tissue: biomaterial development, scaffold fabrication, and tissue engineering. AIChE J. 2008; 54(12): 3048-67.

[11] Langer R. Biomaterials in drug delivery and tissue engineering: one laboratory's experience. Acc Chem Res. 2000; 33: 94-101.

[12] Hutmacher DW. Scaffolds in tissue engineering bone and cartilage. Biomaterials. 2000; 21: 2529-43. 
[13] Shea LD, Smiley E, Bonadio J, Mooney DJ DNA delivery frompolymer matrices for tissue engineering. Nat Biotechnol. 1999; 17: 551-4.

[14] Hoffman AS. Hydrogels for biomedical applications. Adv Drug Deliv Rev. 2002; 43: 3-12.

[15] Drury JL, Mooney DJ. Hydrogels for tissue engineering: scaffold design variables and applications. Biomaterials. 2003; 24: 4337-51.

[16] Lyons F, Partap S, O'Brien FJ. Part 1: scaffolds and surfaces. Technol Health Care. 2008; 16: 305-17.

[17] Khang G, Lee SJ, Kim MS, Lee HB. Biomaterials: tissue engineering and scaffold. In Webster $\mathrm{J}$ (ed.). Encyclopedia of Medical Devices and Instrumentation, Vol 2; 2006: 366-83.

[18] Chung HJ, Park TG. Surface engineered and drug releasing pre-fabricated scaffolds for tissue engineering. Adv Drug Deliv Rev. 2007;59:249-59.

[19] Karande ST, Agrawal MC. Functions and requirement of synthetic scaffolds in tissue engineering. In: Laurencin CT, Nair LS (eds.). Nanotechnology and Tissue Engineering: The Scaffolds; 2008:53.

[20] Ma PX. Biomimetic materials for tissue engineering. Adv Drug Deliv Rev. 2008; 60:184-98.

[21] Drury JL, Mooney DJ. Hydrogels for tissue engineering: scaffold design variablesand applications. Biomaterials. 2003; 24: 4337-51.

[22] Hoffman AS. Hydrogels for biomedical applications. Adv Drug Deliv Rev. 2002; 43: 3-12.

[23] Augst AD, Kong HJ, Mooney DJ. Alginate hydrogels as biomaterials. Macromol Biosci. 2006; 6: 623-33.

[24] Huang S, Fu X. Naturally derived materials-based cell and drug delivery systems in skin regeneration. J Control Release. 2010;142:149-59.

[25] Park CJ, Clark SG, Lichtensteiger CA, Jamison $\mathrm{RD}$, Johnson AJ. Accelerated wound closure of pressure ulcers in aged mice by chitosan scaffolds with and without bFGF. Acta Biomater. 2009; 5: 1926-36.

[26] Clark RAF, Singer AJ. Wound repair: basic biology to tissue engineering. London: Academic Press; 2000.

[27] Bradley M, Cullum N, Nelson EA, Petticrew M, Sheldon T, Torgerson D. Systematic reviews of wound care management: (2). Dressings and topical agents used in the healing of chronic wounds. Health Technol Assess. 1999; 3: 1-35.

[28] Lee JE, Park JC, Lee HK, Oh SH, Suh H. Laminin modified infection-preventing collagen membrane containing silver sulfadiazine-hyaluronan microparticles. Artif Organs. 2002; 26: 521-8.

[29] Costa PF. Bone tissue engineering drug delivery. Current Molecular Biology Reports. 2015; 1: 87-93.
[30] Yao Q, Liu Y, Selvaratnam B, Ranjit T. Koodali D, Sun H. Mesoporous Silicate Nanoparticles/3D Nanofibrous Scaffold-mediated Dual-drug Delivery for Bone Tissue Engineering. doi:10.1016/j. jconrel.2018.04.011

[31] Agila S. Poornima J. Magnetically Controlled Nano-Composite Based 3D Printed Cell Scaffolds As Targeted Drug Delivery Systems For Cancer Therapy. Proceedings of the $15^{\text {th }}$ IEEE International Conference on Nanotechnology July 27-30, 2015, Rome, Italy.

[32] Dai J, Jin J, Yang S and Li G. Doxorubicin-loaded PLA/pearl electrospun nanofibrous scaffold for drug delivery and tumor cells treatment. Mater. Res 2017. Express, at press: https://doi.org/10.1088/20531591/aa7479

[33] Bagó J R., Pegna G J., Okolie O, Mohiti-Asli M, Loboa E G, Hingtgen S D. Electrospun nanofibrous scaffolds increase the efficacy of stem cell-mediated therapy of surgically resected glioblastoma DOI: 10.1016/j.biomaterials.2016.03.008

[34] Bao H, Zhang Q, Xu H, Yan Z. Effects of nanoparticle sizeonantitumoractivity of 10-hydroxycamptothecinconjugated gold nanoparticles: in vitro and in vivo studies. International Journal of Nanomedicine 2016:11 929-940.

[35] Chaudhari AA, Vig K, Baganizi DR, Sahu R, Dixit $S$, Dennis V et al. Future Prospects for Scaffolding Methods and Biomaterials in Skin Tissue Engineering: A Review. Int. J. Mol. Sci. 2016, 17, 1974; doi:10.3390/ijms17121974.

[36] Grigore ME. Drug Delivery Systems in Hard Tissue Engineering. SF J Biotechnol Biomed Eng. 2018; 1(1): 1001.

[37] Garg T, Singh O, Arora S \& Murthy RSR. Scaffold: A Novel Carrier for Cell and Drug Delivery. Critical Reviews $^{\mathrm{TM}}$ in Therapeutic Drug Carrier Systems 2012; 29(1): 1-63.

[38] Jaisinghani A, Gupta A, Raj AT. The potential use of scaffold-mediated local delivery of bone modulators in accelerated orthodontics: A hypotheses. Medical Hypotheses 2018, doi: https://doi.org/10.1016/j. mehy. 2018.07.005.

[39] Aliperta, R, Welzel PB, Bergmann R, Freudenberg $\mathrm{U}$, Berndt N, Feldmann A et al. Cryogel-supported stem cell factory for customized sustained release of bispecific antibodies for cancer immunotherapy. Sci. Rep. 7, 42855; doi: 10.1038/srep42855 (2017).

[40] Lokteva A, Haberkorna U, Mier W. Multicyclic Peptides as Scaffolds for the Development of Tumor Targeting Agents. Current Medicinal Chemistry, 2016. 